Case Report

\title{
Emergency Repair of Acute Aortic Dissection in a Patient with Relapsing Polychondritis
}

\author{
Mitsuru Iida, $\mathrm{MD}, \mathrm{PhD},{ }^{1}$ Yukihiko Orime, $\mathrm{MD}, \mathrm{PhD},{ }^{1}$ Tomofumi Umeda, $\mathrm{MD}, \mathrm{PhD},{ }^{1}$ \\ Yusuke Ishii, $\mathrm{MD},{ }^{1}$ and Motomi Shiono, $\mathrm{MD}, \mathrm{PhD}^{2}$
}

\begin{abstract}
Relapsing polychondritis (RP) is a rare, inflammatory, multiorgan disorder affecting cartilaginous structure and other connective tissue. Cardiovascular complications occur in $10 \%-52 \%$ of patients. We report a case of emergency surgery for an acute aortic dissection in a patient with RP. A 45-year-old female who had been taking corticosteroids ( $10 \mathrm{mg} / \mathrm{day}$ ) for 2 years for RP presented at another hospital with dyspnea and severe chest and back pain. Acute aortic dissection was diagnosed, and we performed emergency replacement of the ascending aorta. We could not control the bleeding from suture holes of the native aorta because the vessel was so fragile. Thus, we performed a delayed sternal closure. The day after surgery, bleeding had decreased, and we could close the chest wall. Infection was well controlled, and the patient suffered minimal additional complications. She was discharged to home by herself 29 days after surgery and returned to normal life.
\end{abstract}

Keywords: acute aortic dissection, relapsing polychondritis, emergency operation, delayed sternal closure

\section{Introduction}

Relapsing polychondritis (RP) is a rare, inflammatory, multiorgan disorder affecting cartilaginous structure and other connective tissue. RP was first described in 1923 by Jaksch-Wartenhorst. ${ }^{1)}$ Cardiovascular complications are also known to occur, ${ }^{2)}$ and surgical cases have been reported. ${ }^{2,3)}$ Here, we report a successful surgical case of acute aortic dissection with delayed sternal closure ${ }^{4)}$ in a patient with RP who had been taking oral corticosteroids.

${ }^{1}$ Department of Cardiovascular Surgery, Surugadai Nihon University Hospital, Tokyo, Japan

${ }^{2}$ Department of Cardiovascular Surgery, Nihon University School of Medicine, Tokyo, Japan

Received: January 11, 2012; Accepted: March 5, 2012

Corresponding author: Mitsuru Iida, MD, PhD. Department of Cardiovascular Surgery, Surugadai Nihon University Hospital, 1-8-13, Kandasurugadai, Chiyoda-ku, Tokyo 101-8309, Japan Email: mitsuru@med.nihon-u.ac.jp

(C)2012 The Editorial Committee of Annals of Thoracic and Cardiovascular Surgery. All rights reserved.

\section{Case Report}

A 45-year-old female presented at the emergency department of another hospital with dyspnea and severe chest and back pain at work. She was diagnosed with acute aortic dissection (Stanford type A) and was transferred to our hospital for emergency surgery. She had a history of RP treated with corticosteroids (10 mg/day) for the previous 2 years. Computed tomography (CT) on admission showed acute aortic dissection (Stanford type A). The dissection started at the right coronary artery and continued to the infrarenal abdominal aorta (Fig. 1). No other medical history was available at the time of admission. We performed emergency surgery. Through a median sternotomy, the dissected, ascending aorta was reconstructed by end-to-end anastomosis with a vascular graft (Triplex $28 \mathrm{~mm}$, Terumo Corporation, Tokyo, Japan) using the less invasive quick replacement (LIQR) technique. ${ }^{5)}$ Stump plasty was performed with a felt strip sandwiched in the distal and proximal ends. Withdrawal from extracorporeal circulation was uneventful, but bleeding from the needle holes in the native aorta was uncontrollable. As a result of bleeding problem, we could 

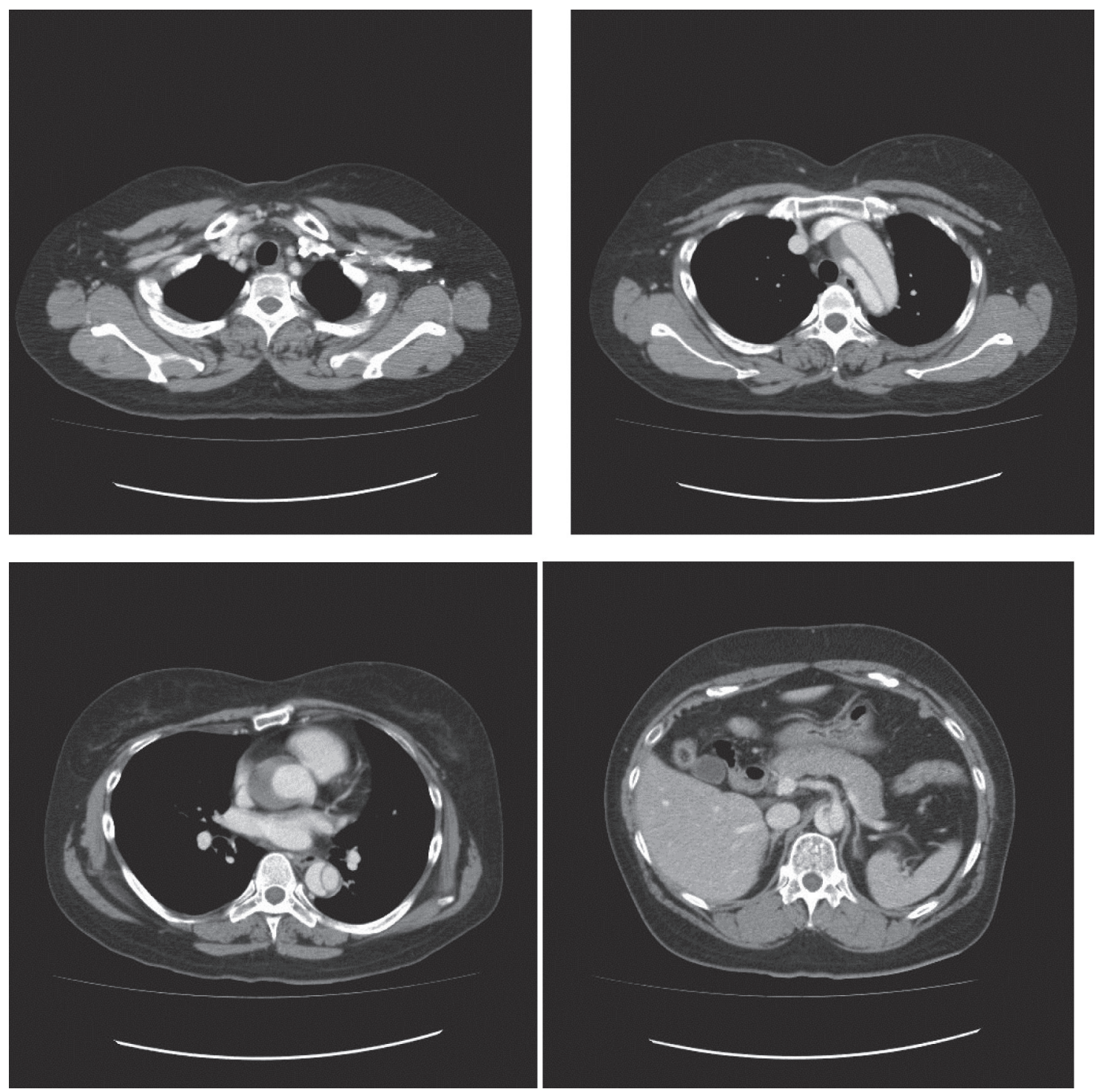

Fig. 1 Computed tomography on admission.

Computed tomography showed Stanford type A acute aortic dissection. The dissection started at the right coronary artery and continued to the infrarenal abdominal aorta.

not close the sternum and decided to use delayed sternal closure. The day after surgery, the bleeding subsided, and inspection of the aorta revealed that the needles holes were no longer bleeding. Thus, it was possible to close the sternum. At that time, the hematoma in the pericardial sac was removed as much as possible, and the wound was sutured with nylon to reduce the risk of subsequent infections. On the following day (postoperative day 2), the patient was extubated and oral intake and steroid therapy was resumed. Infection was well controlled. In the pathological findings of aortic tissue, vascular smooth muscle, arranged roughly, and accumulation of polymorphonu- clear leukocytes, as seen in the hematoxylin-eosin staining, were the findings suggestive of cystic medial necrosis (Fig. 2A and 2B). Furthermore, findings of the toluidine blue staining showed that the vascular fragility accumulation of mucopolysaccharides was observed strongly (Fig. 2C). However, findings suggestive of vasculitis due to an immunological mechanism, such as the infiltration of lymphocytes seen in the RP, were not observed. On postoperative day 29, the patient was discharged alone, travelling on foot to her house. As of the last follow-up at 6 postoperative months, the patient remained well. 

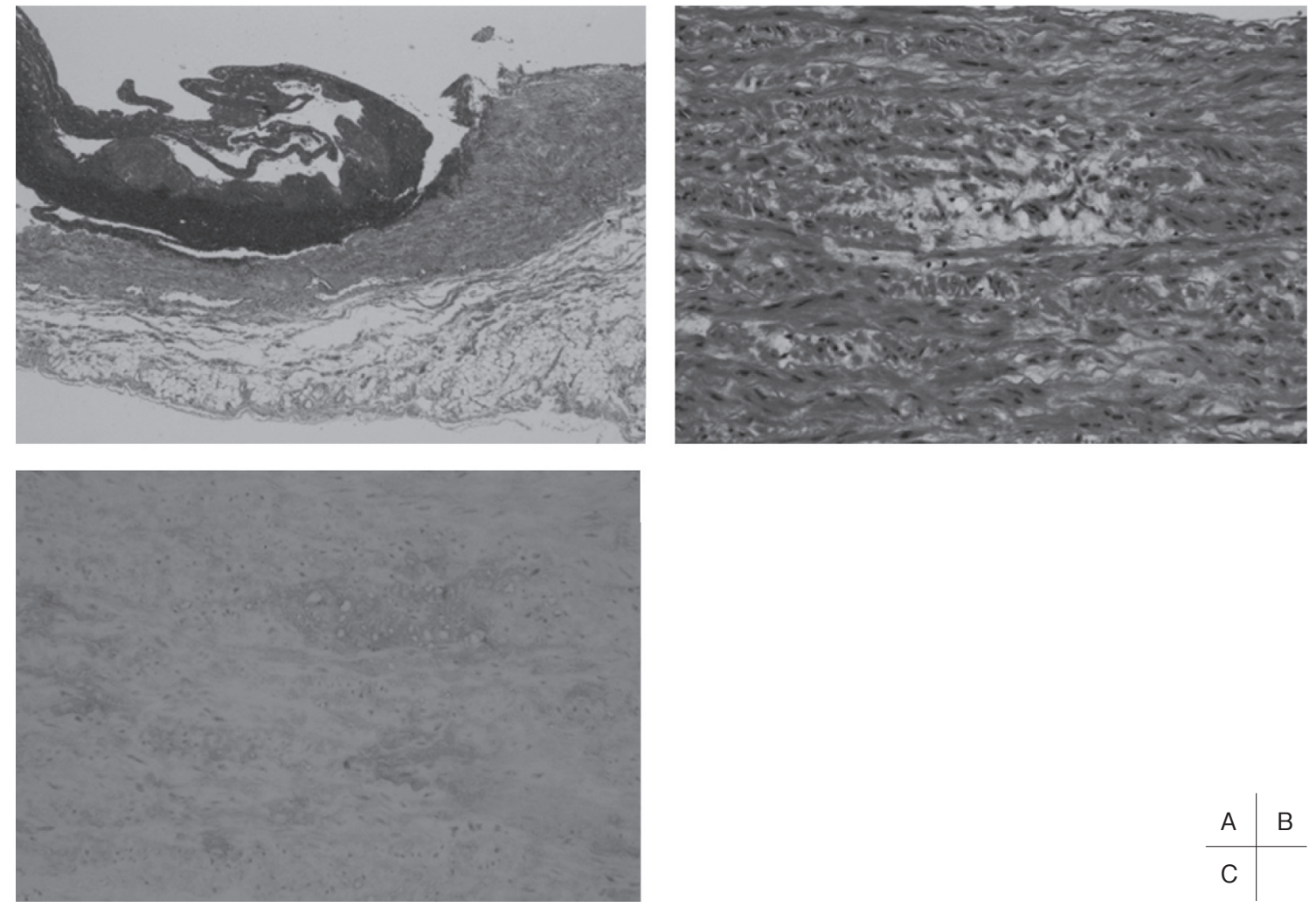

Fig. 2 Pathological findings of aortic tissue.

A, B) Pathological findings of the aortic wall showed a rough arrangement of vascular smooth muscles and accumulation of polymorphonuclear leukocytes.

C) The findings of toluidine blue staining showed that the vascular fragility accumulation of mucopolisaccharides was observed strongly.

\section{Discussion}

$\mathrm{RP}$ is an autoimmune disease that affects the cartilaginous structure and other connective tissue; onset is usually first noted in auricular cartilage. Other affected structures include the nasal septal cartilage, tracheal cartilage, and periocular tissue. Involvement of these structures can lead to manifestations, such as auritis (i.e., pain, redness, and/or deformity of the auricles), saddle nose, soreness of the nose, conjunctivitis, and scleritis. Prognosis is poor, with possible visual complications or loss, cardiovascular problems, and inflammatory conditions of the laryngeal, tracheal, and bronchial cartilage sometimes leading to progressive airway obstruction. Early diagnosis and treatment is very important to reduce the severity of these complications.

Previous reports have described cardiovascular complications of RP. The first such report was of aortic valve regurgitation in 1966, by Yamazaki, ${ }^{6}$ ) and the first reported cardiac valve replacement in a patient with RP was in 1967. ${ }^{2)}$ Postoperative prognosis was not so good because surgery caused disruption of the elastic fibers. The prevalence of cardiovascular disease in persons with RP reportedly ranges from $10 \%$ to $51 \%$. $^{3,7)}$ In addition to aortic valve regurgitation, cardiovascular complications include mitral valve regurgitation, pericarditis, aortic aneurysm, and conduction disturbance. Prognosis after surgery for these other disorders remains poor, because of infection (mediastinitis and overwhelming sepsis) and cardiac events. $^{2,3,8)}$

In our review of previous reports, we found only one case of ascending aorta replacement ${ }^{3)}$ and one case of aortic root replacement. ${ }^{8)}$ In these patients, corticosteroid therapy contributed to aortic tissue fragilization that resulted in suture leakage and prosthesis dehiscence. Our patient's acute aortic dissection required emergency surgery, and she had already been taking corticosteroids for 2 years. As a result, we could not control the bleeding from the aortic suture holes and had to use delayed sternal closure. This allowed us to ensure that she was 
hemodynamically stable before sternal closure. In addition, corticosteroids raised the risk of asymptomatic infection. Oral steroids upon the fragile of connective tissue disease, the difficulty of surgery is very high. Especially of emergency surgery, the surgeon will place more stress. The preoperative informed consent is very important, a fully carried out description of the risks. Fortunately, she recovered from surgery and was able to return home 29 days later. Thus, in her case, surgery was a lifesaving measure. Given the poor prognosis associated with RP and related cardiac surgery, the needs of each patient must be considered when recommending and proceeding with surgery.

\section{Disclosure Statement}

The authors have no conflict of interest to disclose with respect to this presentation.

\section{References}

1) Jaksch-Wartenhorst R. Polychondropathia. Wien Arch F Inn Med 1923; 6: 93-100.

2) Pappas G, Johnson M. Mitral and aortic valvular insufficiency in chronic relapsing polychondritis. Arch Surg 1972; 104: 712-4.

3) Dib C, Moustafa SE, Mookadam M, et al. Surgical treatment of the cardiac manifestations of relapsing polychondritis: overview of 33 patients identified through literature review and the Mayo Clinic records. Mayo Clin Proc 2006; 81: 772-6.

4) Fanning WJ, Vasko JS, Kilman JW. Delayed sternal closure after cardiac surgery. Ann Thorac Surg 1987; 44: 169-72.

5) Hata M, Suzuki M, Sezai A, et al. Less invasive quick replacement for octogenarians with type A acute aortic dissection. J Thorac Cardiovasc Surg 2008; 136: 48993.

6) Yamazaki Y, Yawata K, Hannya H, et al. A case of relapsing polychondritis associated with aortic insufficiency. Jpn Heart J 1966; 7: 188-95.

7) Edrees A. Relapsing polychondritis: a description of a case and review article. Rheumatol Int 2011; 31: 70713.

8) Lang-Lazdunski L, Pansard Y, Hvass U. Aortic valve replacement in relapsing polychondritis. J Thorac Cardiovasc Surg 1997; 114: 131-2. 